LIV. Collision of a Particles with Light Atoms. IV. An Anomalous Effect in Nitrogen. By Professor Sir E. Rutherford, F.R.S.*

T has been shown in paper I. that a metal source, conted with a deposit of radium $\mathrm{C}$, always gives rise to a number of scintillations on a zinc sulphide screen far beyond the range of the $\alpha$ particles. The swift atoms causing these scintillations carry a positive charge and are deflected by a magnetic field, and have about the same range and energy as the swift $\mathrm{H}$ atoms produced by the passage of $\alpha$ particles through hydrogen. These "natural" scintillations are believed to be due mainly to swift $\mathrm{H}$ atoms from the radioactive source, but it is difficult to decide whether they are expelled from the radioactive source itself or are due to the action of $\alpha$ particles on occluded hydrogen.

The apparatus employed to study these " natural" scintillations is the same as that described in paper I. The intense source of radium (1 was placed inside a metal box about $3 \mathrm{~cm}$. from the end, and an opening in the end of the box was covered with a silver plate of stopping power equal to about $6 \mathrm{~cm}$. of air. The zinc sulphide screen was mounted outside, about $1 \mathrm{~mm}$. distant from the silver plate, to admit of the introduction of absorbing foils between them. The whole apparatus was placed in a strong magnetic field to deflect the $\beta$ rays. The variation in the number of these "natural" scintillations with absorption in terms of ems. of air is shown in fig. 1 , curve $A$. In this case, the air in the box was exhausted and absorbing foils of aluminium were used. When dried oxygen or carbon dioxide was admitted into the vessel, the number of scintillations diminished to about the amount to be expected from the stopping power of the column of gas.

A surprising effect was noticed, however, when dried air was introduced. Instead of diminishing, the number of scintillations was increased, and for an absorption corresponding to about $19 \mathrm{~cm}$. of air the number was about twice that observed when the air was exhausted. It was clear from this experiment that the $\alpha$ particles in their passage through air gave riss to long-range scintillations which appeared to the eye to be about equal in brightness to $\mathrm{H}$ scintillations. A systematic series of observations was undertaken to account for the origin of these scintillations. In the first place we have seen that the passage of $\alpha$ particles through nitrogen and

* Communicated by the Author. 
oxygen gives rise to numerous bright scintillations which have a range of about $9 \mathrm{~cm}$. in air. These scintillations have about the range to be expected if they are due to swift $\mathrm{N}$ or $\mathrm{O}$ atoms, carrying unit charge, produced by collision with $\alpha$ particles.

Fig. 1.

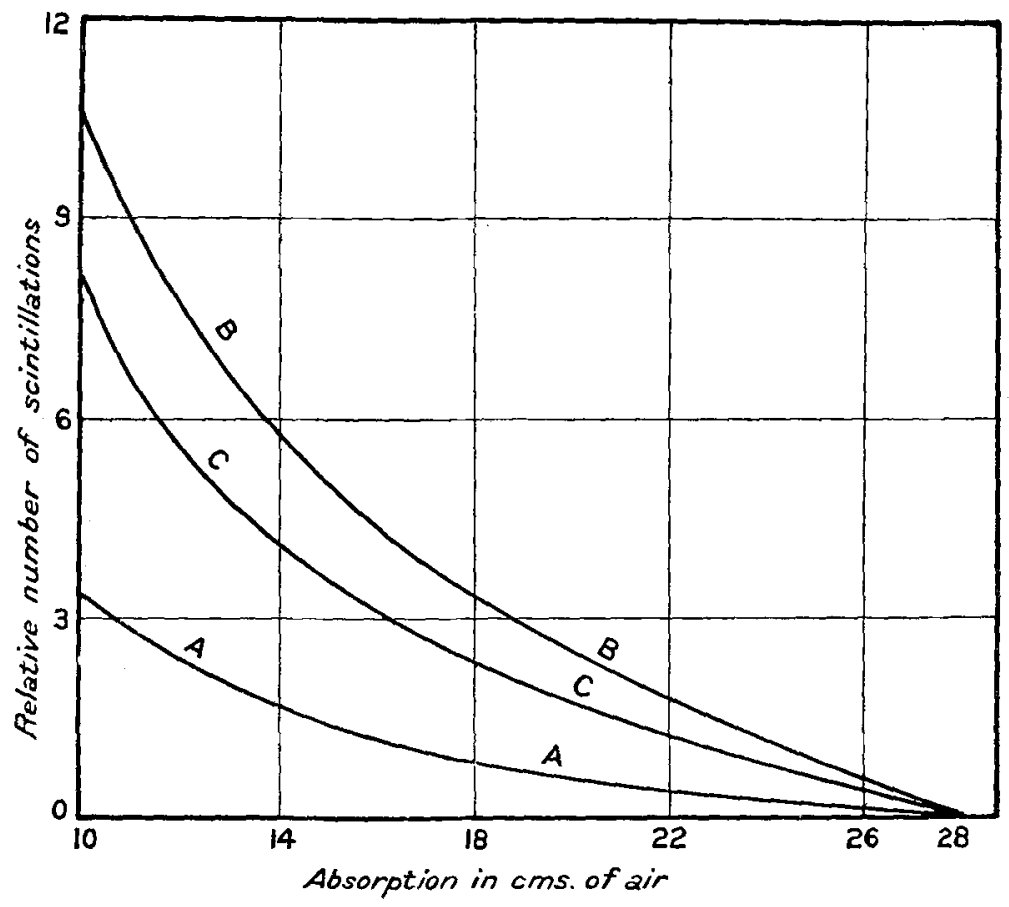

All experiments have consequently been made with an absorption greater than $9 \mathrm{~cm}$. of air, so that these atoms are completely stopped before reaching the zine sulphide screen.

It was found that these long-range scintillations could not be due to the presence of water vapour in the air; for the number was only slightly reduced by thoroughly drying the air. This is to be expected, since on the average the number of the additional scintillations due to air was equivalent to the number of $H$ atoms produced by the mixture of hydrogen at $6 \mathrm{~cm}$. pressure with oxygen. Since on the average the vapour pressure of water in air was not more than $1 \mathrm{~cm}$, the effects of complete drying would not reduce the number by more than one sixth. Even when oxygen and carbon dioxide saturated with water vapour at $20^{\circ} \mathrm{C}$. 
were introduced in place of dry air, the number of scintillations was much less than with dry air.

It is well known that the amount of hydrogen or gases containing hydrogen is normally very small in atmospheric air. No difference was observed whether the air was taken directly from the room or from outside the laboratory or was stored for some days over water.

There was the possibility that the effect, in air night be due to liberation of $\mathrm{H}$ atoms from the dust nuclei in the air. No appreciable difference, however, was observed when the dried air was filtered through long plugs of cotton-wool, or by storage over water for some days to remove dust nuclei.

Since the anomalous effect was observed in air, but not in oxygen, or carbon dioxide, it must be due either to nitrogen or to one of the other gases present in atmospheric air. The latter possibility was excluded by comparing the effects produced in air and in chemically prepared nitrogen. The nitrogen was obtained by the well-known method of adding ammonium chloride to sodium nitrite, and stored over water. It was carefully dried before admission to the apparatus. With pure nitrogen, the number of long-range scintillations under similar conditions was greater than in air. As a result of careful experiments, the ratio was found to be $1 \cdot 25$, the value to be expected if the scintillations are due to nitrogen.

The results so far obtained show that the long-range scintillations obtained from air must be ascribed to nitrogen, but it is important, in addition, to show that they are due to collision of a particles with atoms of nitrogen through the volume of the gas. In the first place, it was found that the number of the scintillations varied with the pressure of the air in the way to be expected if they resulted from collision of $\alpha$ particles along the column of gas. In addition, when an absorbing screen of gold or aluminium was placed close to the source, the range of the scintillations was found to be reduced by the amount to be expected if the range of the expelled atom was proportional to the range of the colliding \& particles. These results show that the scintillations arise from the volume of the gas and are not due to some surface effect in the radioactive source.

In fig. 1 curve $A$ the results of a typical experiment are given showing the variation in the number of natural scintillations with the amount of absorbing matter in their path measured in terms of centimetres of air for $\alpha$ particles. In these experiments carbon dioxide was introduced at a pressure calculated to give the same absorption of the $\alpha$ rays as ordinary air. In curve $B$ the corresponding curve is given when air 
at N.T.P. is introduced in place of carbon dioxide. The difference curve $\mathrm{C}$ shows the corresponding variation of the number of scintillations arising from the nitrogen in the air. It was generally observed that the ratio of the nitrogen effect to the natural effect was some what greater for $19 \mathrm{~cm}$. than for $12 \mathrm{~cm}$. absorption.

In order to estimate the magnitude of the effect, the space between the source and screen was filled with carbon dioxide at diminished pressure and a known pressure of hydrogen was added. The pressure of the carbon dioxide and of hydrogen were adjusted so that the total absorption of a particles in the mixed gas should be equal to that of the air. In this way it was found that the curve of absorption of $H$ atoms produced under these conditions was some what steeper than curve $C$ of fig. 1. As a consequence, the amount of hydrogen mixed with carbon dioxide required to produce a number of scintillations equal to that of air, increased with the increase of absorption. For example, the effect in air was equal to about $4 \mathrm{~cm}$. of hydrogen at $12 \mathrm{~cm}$. absorption, and about $8 \mathrm{~cm}$. at $19 \mathrm{~cm}$. absorption. For a mean value of the alsorption, the effect was equal to about $6 \mathrm{~cm}$. of hydrogen. This increased absorption of $H$ atoms under similar conditions indicated either that (1) the swift atoms from air had a somewhat greater range than the $\mathrm{H}$ atoms, or (2) that the atoms from air were projected more in the line of flight of the $\alpha$ particles.

While the maximum range of the scintillations from air nsing radium $\mathrm{C}$ as a source of $a$ rays appeared to be about the same, viz. $28 \mathrm{~cm}$., as for $\mathrm{H}$ atoms produced from hydrogen, it was difficult to fix the end of the range with certainty on account of the smallness of the number and the weakness of the scintillations. Some special experiments were made to test whether, under favourable conditions, any scintillations due to nitrogen could be observed beyond $28 \mathrm{~cm}$. of air absorption. For this purpose a strong source (about $60 \mathrm{mg}$. Ra activity) was brought within $2 \cdot 5 \mathrm{~cm}$. of the zinc sulphide screen, the space between containing dry air. On still further reducing the distance, the screen hecame too bright to detect very feeble scintillations. No certain evidence of scintillations was found beyond a range of $28 \mathrm{~cm}$. It would therefore appear that (2) above is the more probable explanation.

In a previons paper (III.) we have seen that the number of swift atoms of nitrogen or oxygen produced per unit path by collision with $a$ particles is about the same as the corresponding number of $\mathrm{H}$ atoms in hydrogen. Since the number of long-range scintillations in air is equivalent to that produced under similar conditions in a column of hydrogen at $6 \mathrm{~cm}$. 
pressure, we may consequently conclude that only one longrange atom is produced for every 12 close collisions giving rise to a swift nitrogen atom of maximum range $9 \mathrm{~cm}$.

It is of interest to give data showing the number of longrange scintillations produced in nitrogen at atmospheric pressure under definite conditions. For a column of nitrogen $3.3 \mathrm{~cm}$. long, and for a total absorption of $19 \mathrm{~cm}$. of air from the source, the number due to nitrogen per milligram of activity is $\cdot 6$ per minute on a screen of $3 \cdot 14 \mathrm{sq}$. mm. area.

Both as regards range and brightness of scintillations, the long-range atoms from nitrogen closely resemble $H$ atoms, and in all probability are luddrogen atoms. In order, however, to settle this importint point definitely, it is necessary to determine the detlexion of these atoms in a magnetic field. Some preliminary experiments have been made by a method similar to that employed in measuring the velocity of the $H$ atom (see paper II.). The main lifficulty is to obtain a sufficiently large deflexion of the stream of atoms and yet have a sufficient number of scintillations per minute for counting. The a rays from a strong source passed through dry air between two parallel horizontal plates $3 \mathrm{~cm}$. long and $1.6 \mathrm{~mm}$. apart, and the number of scintillations on the screen placed near the end of the plates was observed for different strengths of the magnetic field. Under these conditions, when the scintillations arise from the whole length of the column of air between the plates, the strongest magnetic field available reduced the number of scintillations by only 30 per cent. When the air was replaced by a mixture of carbon dioxide and hydrogen of the same stopping power for a rays, about an equal reduction was noted. As tar as the experiment goes, this is an indication that the scintillations are due to $\mathrm{H}$ atoms; but the actual number of scintillations and the amount of reduction was too small to place much reliance on the result. In order to settle this question definitely, it will probably prove necessary to employ a solid nitrogen compound, free from hydrogen, as a source, and to use much stronger sources of a rays. In snch experiments, it will be of importance to discriminate between the deflexions due to $\mathrm{H}$ atoms and possible atoms of atomic weight 2. From the calculations given in paper III., it is seen that a collision of an a particle with a free atom of mass 2 should give rise to an atom of range about $32 \mathrm{~cm}$. in air, and of initial energy about 89 of that of the $H$ atom produced under similar conditions. The deflexion of the pencil of these rays in a magnetic field should be about 6 of that shown by a corresponding pencil of $\mathrm{H}$ atoms.

Phil. Mag. S. 6. Vol. 37. No. 222. June 1919. 2 S 


\section{Discussion of results.}

From the results so far obtained it is difficult to avoid the conclusion that the long-range atoms arising from collision of $\alpha$ particles with nitrogen are not nitrogen atoms but probabiy atoms of hydrogen, or atoms of mass 2 . If this be the case, we must conclude that the nitrogen atom is disintegrated under the intense forces developed in a close collision with a swift $\alpha$ particle, and that the hydrogen atom which is liberated formed a constituent part of the nitrogen nucleus. We have drawn attention in paper III. to the rather surprising observation that the range of the nitrogen atoms in air is about the same as the oxygen atoms, although we should expect a difference of abont 19 per cent. If in collisions which give rise to swift nitrogen atoms, the hydrogen is at the same time disrupted, such a difference might be accounted for, for the energy is then shared between two systems.

It is of interest to note, that while the majority of the light atoms, as is well known, have atomic weights represented by $4 n$ or $4 n+3$ where $n$ is a whole number, nitrogen is the only atom which is expressed by $4 n+2$. We should anticipate from radioactive data that the nitrogen nucleus consists of three helium nuclei each of atomic mass 4 and either two hydrogen nuclei or one of mass 2 . If the $\mathrm{H}$ nuclei were outriders of the main system of mass 12, the number of close collisions with the bound $\mathrm{H}$ nuclei would be less than if the latter were free, for the $\alpha$ particle in a collision comes under the combined field of the $H$ nucleus and of the central mass. Under such conditions, it is to be expected that the a particle would only occasionally approach close enough to the $H$ nucleus to give it the maximum velocity, although in many cases it may give it sufficient energy to break its bond with the central mass. Such a point of view would explain why the number of swift $\mathrm{H}$ atoms from nitrogen is less than the corresponding number in free hydrogen and less also than the number of swift nitrogen atoms. The general results indicate that the $\mathrm{H}$ nuclei, which are released, are distant about twice the diameter of the electron $\left(7 \times 10^{-13} \mathrm{~cm}\right.$.) from the centre of the main atom. Without a knowledge of the laws of force at such small distances, it is difficult to estimate the energy required to free the $\mathrm{H}$ nucleus or to calculate the maximum velocity that can be given to the escaping $\mathrm{H}$ atom. It is not to be expected, a priori, that the velocity or range of the $\mathrm{H}$ atom released from the nitrogen atom should be identical with that due to a collision in free hydrogen.

Taking into account the great energy of motion of the a particle expelled from radium $\mathrm{C}$, the close collision of such 
an $\alpha$ particle with a light atom seems to be the most likely agency to promote the disruption of the latter; for the forces on the nuclei arising from such collisions appear to be greater than can be produced by any other agency at present available. Considering the enormous intensity of the forces brought into play, it is not so much a matter of surprise that the nitrogen atom should suffer disintegration as that the $\alpha$ particle itself escapes disruption into its constituents. The results as a whole suggest that, if $\alpha$ particles-or similar projectiles--of still greater energy were available for experiment, we might expect to break down the nucleus structure of many of the lighter atoms.

I desire to express my thanks to Mr. William Kay for his invaluable assistance in counting scintillations.

University of Manchester, A pril 1919.

\section{The Rotational Oscillation of a Cylinder in a Viscous Liquid. By D. Coster *}

THIs problem has been dealt with by Stokes $\dagger$ for the 1 purpose of numerical calculations to determine the viscosity of the air. Still, I think it interesting to publish another solution of the problem which gives more opportunity of discussing the different cases, thongh it is perhaps less adapted to precise calculations.

The method to be followed will be in the main the same as that used by Prof. Verschaffelt in the analogous case of the sphere $\ddagger$. We consider the rotational swings about its axis of an infinitely long cylinder which executes a forced vibration. Our object will be to ascertain the motion in the liquid which will establish itself after an infinite time (in practice atter a relatively short time $\$$ ) in order to compute tho frictional moment of forces exerted on the cylinder by the liquid. The calculations will be referred to a height of $1 \mathrm{~cm}$.

The motion of the cylinder may be represented by $\alpha=a \cos p t$ where $\alpha$ is the angle of rotation. An obvious assumption to be made is that the liquid will be set in motion in coaxial cylindrical shells each of wbich. will execute its oscillations as a whole. On this assumption it is not difficult

* Communicated by Prof. G. N. Watson, M.A., D.Sc. First published in the Amsterdam Proc. May 1918, vol. xxi. p. 193 ,

+ Math. Papers, vol. v. p. 207.

$\pm C f$. Amst. Proc. vol. xviii. p. 840 ; Comm. Leiden, $148 \mathrm{C}$.

$\S$ Cf. Comm. Leiden, p. 22, fiotnote. 